\title{
LUMINOUS INFRARED GALAXIES IN A MERGING SEQUENCE: ISO OBSERVATIONS
}

\author{
C.-Y. HWANG AND K.Y. LO \\ Institute of Astronomy and Astrophysics, Academia Sinica \\ P.O. Box 1-87, Nankang, Taipei, Taiwan 115, R.O.C. \\ Y. GAO AND R.A. GRUENDL \\ Department of Astronomy, University of Illinois, U.S.A. \\ AND \\ N.-Y. LU \\ IPAC, Caltech, U. S. A.
}

We report mid-infrared images of several luminous infrared galaxies (LIGs) taken with ISOCAM on the Infrared Space Observatory (ISO). These LIGs were chosen to represent different phases of a merger sequence of galaxygalaxy interaction with special emphasis on early/intermediate stages of merging. The molecular gas distribution of these LIGs has also been mapped at high spatial resolution (see contribution by Gao et al., this volume). The goal is to do a synoptic study of the evolution of physical conditions in these LIGs along the merger sequence.

The ISOCAM $15 \mu \mathrm{m}$ (LW3) images of these LIGs show extended structures for the early and intermediate mergers. Individual galaxies are resolved, and the distribution of the mid-infrared emission has similar morphology as that of $\mathrm{CO}$ emission (see Gao et al., this volume); the results indicate that most of the IR luminosity of these LIGs is not from a central AGN. The ratios of the peak fluxes to the total fluxes of the $15 \mu \mathrm{m}$ emission of these LIGs increase from around 0.05 for the early mergers to around 0.1 for the advanced mergers except for UGC 2369 and Mrk 848, which were found to have undergone some other merging processes. It is noted that there is a similar trend for the star formation efficiency $\left(L_{\mathrm{IR}} / M\left(\mathrm{H}_{2}\right)\right.$; $\mathrm{SFE}$ ), which also increases roughly by a factor of two from the early mergers to the intermediate/advanced mergers for these LIGs (see Gao et al., this volume). 